\title{
Parkinson's disease and the frequent reasons for emergency admission
}

\author{
Ozlem Guneysel' \\ Oguzhan Onultan ${ }^{2}$ \\ Ozge Onur'
}

'Marmara University School of Medicine, Department of Emergency Medicine, Istanbul, Turkey; ${ }^{2}$ Kadıköy Universal Hospital, Department of Neurology, Istanbul, Turkey
Correspondence: Ozlem Guneysel Marmara University School Of Medicine, Department of Emergency Medicine, Tophanelioglu CYurtacan S No I3-15. Altunizade/lstanbul, Turkey

Tel +90532 22I 1368

Fax +902163269578

Email guneysel@gmail.com

\begin{abstract}
Introduction: Available data suggest that Parkinson's disease (PD) patients have a significant socioeconomic impact owing partly to increased hospital and drug utilization. The aims of this study were to provide a profile of patients with PD who required admission to hospital and to determine the reasons for emergency admission.

Patients and methods: Between September 1st, 2004 and August 31st, 2006, patients with PD who were admitted to our emergency department (ED) were included in the study. Patients with PD who were diagnosed by a neurologist formerly, and admitted to the ED with any reason constituted the study population. Demographical data, reasons for admission, years exposed to $\mathrm{PD}$, number of admissions to the emergency department in the past 12 months, prior Hoehn and Yahr (H\&Y) scores were recorded. H\&Y was performed again for all patients 4 weeks after discharge.

Results: Seventy-six patients with PD were included in the study. Reasons for admission to hospital were infectious diseases (31.6\%), trauma (27.6\%), cardiovascular emergencies $(14.5 \%)$, cerebrovascular emergencies $(11.8 \%)$, gastrointestinal emergencies $(7.9 \%)$, and electrolyte disturbances $(6.6 \%)$, respectively. There was no dependence between the time of exposure to $\mathrm{PD}$ and $\mathrm{H} \& \mathrm{Y}$ score. Number of emergency admittance in the last 12 months was independent from the last H\&Y score $(p=0.297)$. However, there was a dependency between the reasons for emergency admittance and the H\&Y scores $(p=0.023)$.

Discussion: H\&Y score is not dependent on the emergency admittance or on the outcome after discharge from the emergency department. The motor disability by itself cannot predict the whole picture of PD and the systemic complications leading to emergency admittance.
\end{abstract}

Keywords: Parkinson's disease, emergency admittance, Hoehn and Yahr scale.

\section{Introduction}

Parkinson's disease (PD) effects approximately 7 in 1000 persons 40 years and older and 3 in 100 persons 80 years and older. Its incidence increases with advancing age (Tanner et al 1992; Bower et al 2000). Available data suggest that patients with PD have a significant socioeconomic impact owing partly to increased hospital and drug utilization. As the worldwide population is aging, we may expect these figures to increase with time (Zhang et al 1993). PD causes significant expense for the national healthcare system due to its chronic progressive course, duration of the disease, the high prevalence, and the devastating prognosis (Dodel et al 1997). The chronic, progressive course of the disease, which often leads to severe disability, results in reduced or lost productivity as a result of illness or premature death (Dodel et al 1998). The disease can be associated with a wide range of complications of advancing disease. However, it is unclear how often these occur in the overall population of patients with PD (Schrag et al 2002).

Patients with PD in the early stages generally do well and do not require emergency treatment. It is in the advanced stages that emergent situations begin to arise (Factor et al 2000). As PD is usually managed within specialty clinics by specialist physicians, 
the nonspecialists often have little experience with this condition. However, at times of emergency admission, the patient is often cared for by nonspecialists. Also, given the specific needs of such patients, unfortunately, patient care is often suboptimal (Woodford et al 2005). The aims of this study were to provide a profile of patients with PD who required admission to hospital, and to determine the reasons for emergency admission.

\section{Patients and methods}

The study was conducted at the Marmara University School of Medicine, Department of Emergency Medicine in Istanbul, Turkey. Our emergency department (ED) sees a volume of approximately 30,000 patients per year. Between September 1st, 2004 and August 31st, 2006, patients with PD admitted to our ED were included in the study. Patients with PD who were diagnosed by a neurologist formerly, and admitted to the ED with any reason constituted the study population. Admissions that resulted in the death of the patient and diagnosis of the PD that identified in the ED for the first time were excluded from the study.

All patients and surrogates were requested to complete a written informed consent and a questionnaire after they were admitted to the ED and stabilized. Demographical data, reasons for admission, hospitalization period (day), years exposed to PD, number of admissions to the ED in the past 12 months were recorded to a data sheet. During the hospital stay all files of patients were searched retrospectively and prior Hoehn and Yahr Scale (H\&Y) scores were recorded. All patients were requested for recheck 4 weeks after discharge and H\&Y was performed once more. Two clinicians, one from the ED and one from neurology department performed the H\&Y on all patients.

\section{Statistical analysis}

Analyses were performed with SPSS version 11.0 (SPSS Inc., Chicago, IL, USA). Chi-square test was performed to compare the parameters. Data were given within $95 \%$ confidence intervals $(\mathrm{CI})$. In the analysis, $\mathrm{P}<0.05$ was statistically significant.

\section{Results}

We obtained information from 76 patients with PD. Mean age was $73.18 \pm 10.16$ years (range 56-96 years) and $36.8 \%$ were female and $63.2 \%$ were male. Reasons for admission to hospital were infectious diseases $(31.6 \%)$, trauma $(27.6 \%)$, cardiovascular emergencies $(14.5 \%)$, cerebrovascular emergencies $(11.8 \%)$, gastrointestinal emergencies $(7.9 \%)$, and
Table I Reasons for emergency hospital admissions

\begin{tabular}{lll}
\hline & $\mathbf{n}$ & $\%$ \\
\hline Infections & 9 & \\
Pneumoniae & 15 & 11.8 \\
UTI & & 19.7 \\
Trauma & 6 & \\
Head & 7 & 7.8 \\
Upper extremity & 8 & 9.2 \\
Lower extremity & & 10.5 \\
Cardiovascular & 9 & \\
Heart failure & 2 & 11.8 \\
Acute coronary syndrome & & 2.6 \\
Cerebrovascular & 8 & 10.5 \\
TIA & 1 & 1.3 \\
ICH & & \\
Gastrointestinal & 4 & 5.2 \\
UPper Gl bleeding & 2 & 2.6 \\
Lower Gl bleeding & 5 & 6.5 \\
Electrolyte disturbances & 76 & 100 \\
TOTAL & & \\
\hline
\end{tabular}

Abbreviations: $\mathrm{Gl}$, gastrointestinal; $\mathrm{ICH}$, intracranial hemorrhage; TIA, transient ischemic attack; UTI, urinary tract infection.

electrolyte disturbances (6.6\%), respectively (Table 1). The mean duration of hospital stay was $12.24 \pm 7.19$ days (range 2-32 days). Mean years exposed to PD were 5.61 \pm 3.73 years (range $1-17$ years). Twenty-one patients $(27.6 \%)$ were doing well and did not require home nursing, and 55 (72.4\%) required home care. Thirty-four patients $(44.7 \%)$ visited the ED once in the past 12 months, 19 patients (25\%) twice, and 23 patients $(30.3 \%)$ three times or more. Fifty-two patients (68.5\%) were using their medications as ordered by the attending neurologist and 24 patients (31.5\%) were not.

No dependence was found between the time (years) exposed to PD and the number of emergency admittance $(p=0.087)$. The number of emergency admittance was independent from regular medicine use $(p=0.263)$. Also, there was no dependence between regular medicine use and the reasons for energency admittance $(p=0.951)$. There was no dependence between the time exposed to PD and $H \& Y$ score $(p=0.246)$. the number of emergency admittance in the last 12 months was independant from the last H\&Y score $(p=0.297)$. However, there was a dependency between the reasons of emergency admittance and the H\&Y score $(p=0.023)$.

\section{Discussion}

This study indicated that the frequency of emergency admittance is not dependent on the duration of PD. According to assumed PD progress in years, we had expected more frequent emergency admittances. This could be explained by three 
factors; first, only major problems (ie, cerebrovascular accident, hip fracture) require admittance to the emergency department; second, late stage PD patients' minor problems are managed at home or caregiving centers by professional visits; and third, the psychological ignorance of caregiving families.

In patients with $\mathrm{PD}$, progressive postural instability causing frequent falls is common. These falls may result in severe head and bodily injury, such as hip fractures. Another problem in late disease is dysphagia leading to aspiration pneumonia or asphyxation. Autonomic dysfunction is also a fairly common cause leading to orthostatic hypotension, bowel and bladder dysfunction. Constipation, fecal impaction, and urinary tract infections are common problems at the later stages of disease. Orthostatic hypotension is not only a disease-related but also a treatment-related disorder. The disease-related component is correlated with disease duration. The treatment-related component is a result of tendency of dopaminergic medications to decrease blood pressure that should be kept in mind (Factor et al 2000).

The H\&Y score is not dependent on the time passed from the onset of $\mathrm{PD}$, at least in our patients. This is also reflected in our study: the H\&Y score is not correlated with the emergency admittance, regarding reason, and outcome parameters. In the study by Giladi and colleagues (2001) festinating gait (FSG), which can be the main reason for falls and fractures, was strongly associated with the H\&Y score and progressed disease as a result, but not with disease severity evaluated by UPDRS. In this study, longer disease duration had been found to be the only clinical factor to be associated with FSG, but not the whole picture. A study by Sato and colleagues (2006) concluded that early onset PD patients showed a longer duration to reach stage III, IV, and V in H\&Y scale, which may be another clue that disease duration and motor disability are not always related.

Emergency admittances of patients with PD are not dependent on their primary disease, but it is indirectly dependent regarding the process of PD. Our study population consisted of clearly defined PD patients and had been evaluated before and after emergency admissions regarding their PD, which was unique. The pioneering reasons for emergency admittance defined in our study was similar to a study done by Woodford and colleagues (2005). In a study by Temlett and colleagues (2006), it was concluded that complications of the later stages of PD and associated treatments are more likely to lead to hospital admission than management of the primary motor disease, which is similar to what we have found in our study as H\&Y scores are not dependant to emergency admissions.
The trauma, cerebrovascular accident and cerebrovascular stroke risks of the patients are not dependent on the patients' PD stage (by H\&Y scale) and the treatment at the ED. In Derejko's study (2006) cardiovascular risk factors had been studied and found not significantly related with motor disability.

The patients with PD start to take medications on a proper schedule when they are in need of caregiving, but this medication does not make any meaningful difference in the frequency of emergency admittance. Meanwhile we cannot predict this for the early stages.

There are unique points we observed in our study. First, the H\&Y scale is not dependent on the disease duration and emergency admittance. Second, the motor disability by itself cannot predict the whole picture of PD and the systemic complications leading to emergency admittance.

\section{Limitations of the study}

The former H\&Y scores which were obtained retrospectively from the files of patients' were performed by another neurologist and not by the authors of this study.

\section{Conclusion}

The reasons for frequent admission to hospital for patients with PD were infectious diseases, trauma, cardiovascular emergencies, cerebrovascular emergencies, gastrointestinal emergencies, and electrolyte disturbances. The H\&Y score is not dependant on the emergency admittance or the outcome after discharge from the emergency department. The motor disability by itself cannot predict the whole picture of PD and the systemic complications leading to emergency admittance.

\section{Disclosure}

The authors report no conflicts of interest.

\section{References}

Bower JH, Maraganore DM, McDonnel SK, et al. 2000. Influence of strict, intermediate and broad diagnostic criteria on the age-and sex- spesific incidence of Parkinson's disease. Mov Disord, 15:819-25.

Derejko M, Slawek M, Wieczorek D, et al. 2006. The influence of vascular risk factors and white matter hyperintensities on the degree of motor impairment in Parkinson's disease. Neurol Neurochir Pol, 40:276-83.

Dodel RC, Eggert KM, Singer MS, et al. 1998. Costs of drug treatment in Parkinson's disease. Mov Disord, 13:249-54.

Dodel RC, Singer M, Kohne-Volland R, et al. 1997. Cost of illness in Parkinson disease. A retrospective 3-month analysis of direct costs. Nervenarzt, 68:978-84.

Factor SA, Molho ES. 2000. Emergency department presentations of patients with Parkinson's disease. Am J Emerg Med, 18:209-15.

Giladi N, Shabtai H, Rozenberg E, et al. 2001. Gait festination in Parkinson's disease. Parkinsonism Relat Disord, 7:135-8. 
Sato K, Hatano T, Yamashiro K, et al. 2006. Prognosis of Parkinson's disease: time to stage III, IV, V, and to motor fluctuations. Mov Disord, 21:1384-95.

Schrag A, Ben-Schlomo Y, Quinn N. 2002. How common are complications of Parkinson's disease? J Neurol Apr, 249:419-23.

Tanner CM, Thelen JA, Offord KP, et al. 1992. Parkinson's disease incidence in Olmsted County, MN: 1935-1988. Neurology, 42(suppl 3):194.
Temlett JA, Thompson PD. 2006. Reasons for Admission to Hospital for Parkinson's Disease. Intern Med J, 36:524-6.

Woodford H, Walker R. 2005. Emergency hospital admissions in idiopathic Parkinson's disease. Mov Disord, 20:1104-8.

Zhang Z, Roman G. 1993. Worldwide occurence of Parkinson's disease: an updated review. Neuroepidemiology, 12:195-208. 